\title{
Maturidade Perceptual e Inteligência
}

Perceptual-motor maturity and intelligence
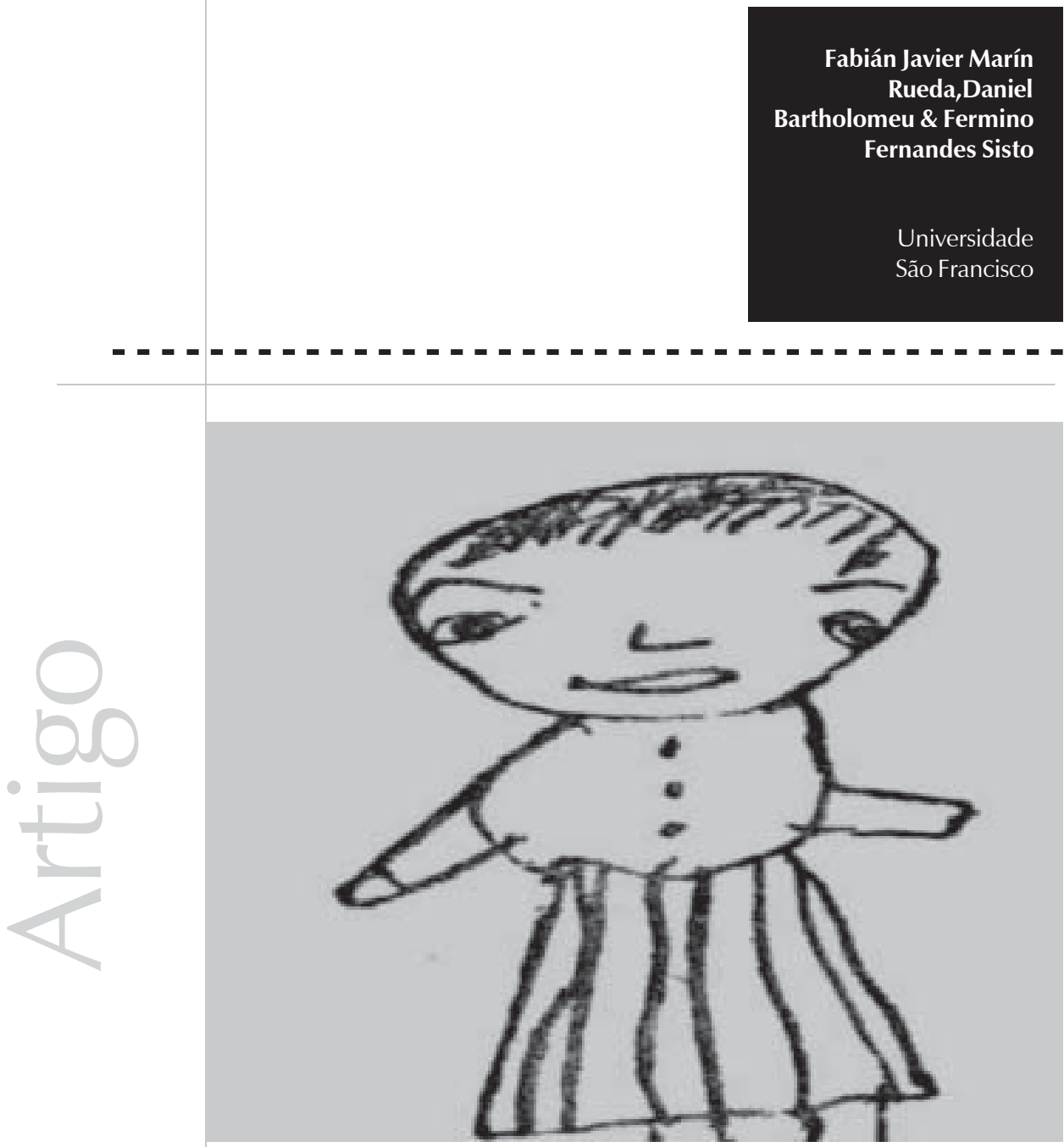


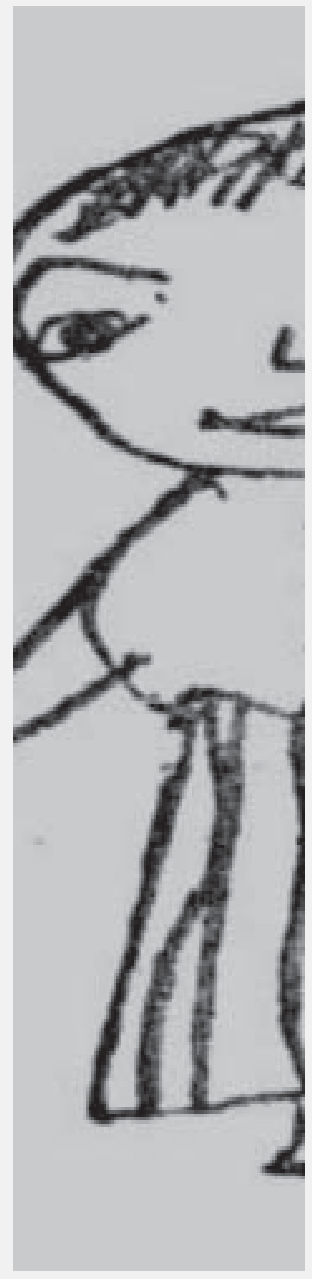

Resumo: O objetivo desta pesquisa foi analisar e correlacionar os resultados obtidos no Teste Guestáltico Visomotor de Bender e no Teste do Desenho da Figura Humana-Goodenough. A hipótese foi que haveria correlação negativa entre os testes, ou seja, conforme aumentasse o nível intelectual das crianças a partir do teste do Desenho da Figura Humana, diminuiria o número de erros apresentados no Bender. Participaram da pesquisa 312 crianças de ambos os sexos, de 1a a 4⿳亠口冋 série, de uma escola pública do interior de São Paulo, com idades variando entre sete e dez anos. Os resultados mostraram correlações negativas e significativas entre os testes, confirmando a hipótese do estudo. Tais resultados permitiriam inferir que o TGB e o DFH estariam medindo parte do mesmo construto, o que poderia ser considerado uma evidência de validade. Palavras-chave: Teste do Desenho da Figura Humana, Teste de Bender, inteligência, instrumentos de medida.

Abstract: The aim of this research was to analyze and correlate the Bender Test and the Human Figure Drawing Test-Goodenough scores. Negative correlation between the tests was hypothesized, that is, as the intellectual level of the children from the Human Figure Drawing Test would increase, the number of errors presented in the Bender Test would decrease. This research investigated 312 children of both genders, from seven to ten years old, attending the $1^{\text {st }}$ to $4^{\text {th }}$ grades of a public school in the State of São Paulo. The results showed negative and significant correlations between the tests, confirming the hypothesis of this study. Such results would allow inferring that the TCB and the DFH would be measuring part of the same construct, what could be considered evidence of validity.

Key words: Human Figure Drawing Test, Bender Test, intelligence, instruments of measure.

Dentre os instrumentos mais relevantes e mais utilizados na avaliação psicológica, encontramse o Teste Gestáltico Visomotor de Bender e o Teste do Desenho da Figura Humana (Alves, 2002; Noronha, 2001, 2002, Noronha e colaboradores, 2002). Sundberg (1961) já apontava o grande uso de ambos os testes, citando como instrumentos psicológicos mais utilizados o Rorschach, o Desenho da Figura Humana (Machover), o TAT, o Teste Gestáltico Visomotor de Bender, o Stanford-Binet, o WAIS e o MMPI, nessa ordem.

O Teste Gestáltico de Bender (TGB) foi criado por Lauretta Bender, em 1938, com o objetivo de fornecer um índice de maturação perceptomotora. Segundo Clawson (1980), Bender ressaltava que a mensuração da maturidade percepto-motora podia ser obtida por meio de princípios de organização gestálticos.

O TGB consta de nove figuras, que são apresentadas uma a uma para a criança copiálas em uma folha em branco. Segundo Koppitz (1989), para Bender, a reprodução das figuras é determinada por princípios biológicos e sensório-motores, que variam em razão do desenvolvimento e nível maturacional do indivíduo, assim como de seu estado patológico funcional e organicamente induzido.

Para Bender (1938, apud Koppitz, 1989) a cópia da gestalt das figuras reflete o nível de maturação da percepção visomotora, que está relacionada com a linguagem e outras funções 
associadas à inteligência, principalmente em crianças pequenas. Dessa forma, o TGB supostamente está relacionado com inteligência nas crianças pequenas, porém, quando a percepção visomotora amadurece por completo, o TGB não é mais indicado como medida de aptidão intelectual. Sendo a idade e a maturação da perceção visomotora fatores determinantes na utilização do TGB como teste de inteligência, Koppitz (1989) realizou um estudo para verificar a correlação da escala de maturação do Bender com a escala de inteligência Stanford-Binet e a escala de inteligência Wechsler para crianças (WISC). Os resultados dessa pesquisa indicaram que o TGB pode ser indicado como medida de inteligência para crianças entre cinco e dez anos, nos Estados Unidos.

Em relação à idade, Koppitz (1989) relata que há um progresso regular na capacidade para copiar os desenhos à medida que aumenta a idade. Porém, isso não significa que as crianças maturam com a mesma velocidade na percepção visomotora, nem que o resultado de cada criança corresponda necessariamente a sua faixa etária. A autora indica ainda que, para crianças até sete anos, o TGB é útil para identificar as imaturas e as que se encontram acima da média; já em crianças de oito anos, o TGB pode detectar aquelas com uma percepção visomotora imatura, fato que também é aplicável às crianças de nove anos, já que, nessa idade, é esperado que a criança possa executar o TGB sem erros sérios.

Deve ser ressaltado que as pesquisas realizadas com o TGB são inúmeras e abarcam diferentes aspectos da avaliação psicológica. Também é importante citar que o tipo de estudo desenvolvido com o TGB variou muito conforme o período em que foi realizado. Nesse sentido, na década de 50 do século passado, foi registrado o maior número de pesquisas do TGB com pacientes psiquiátricos. Assim sendo, o TGB foi pesquisado em pacientes esquizofrênicos (Bambaren, 1958; Matchabely \& Bertrand, 1953), em pacientes psicóticos (Prado, Peyman \& Lacey, 1960;
Stewart \& Cunningham, 1958), em pacientes maníaco-depressivos (Murray \& Roberts, 1956) e comparando pacientes psicóticos com neuróticos (Tamkin, 1957, por exemplo). As décadas de 80 e 90 do século passado mostram o maior número de estudos do TGB com sujeitos adultos que não apresentavam nenhum tipo de distúrbio psiquiátrico ou lesão cerebral (Beutler, 1985; Holmes, Dungan \& Medlin, 1984; Marek, Zajac, Rola \& Wocka, 1995; Okasha, 1985; Sangster, Rogers \& Searight, 1993, entre outros).

Com base na grande quantidade de estudos realizados com ele, assim como na abrangência psicológica de suas pesquisas, percebe-se a importância do TGB na realização da avaliação psicológica. Essa importância também pode ser verificada quando se fala do Teste do Desenho da Figura Humana (DFH) e suas implicações. Os estudos com o DFH abarcam diferentes áreas da avaliação psicológica. Nesse sentido, Kahill (1984) e Curty (1985) evidenciaram que o DFH é uma das técnicas psicológicas mais utilizadas. Nguyen (1992) confirma os resultados anteriores, ressaltando que o desenho infantil mais freqüentemente utilizado para medir a inteligência das crianças é o DFH. O DFH também já foi relacionado às dificuldades de aprendizagem (Bachara, Zaba \& Raskin, 1975; Gounard \& Pray, 1975; Jing, Yuan \& Liu, 1999; Pollak, 1986), ao desenvolvimento cognitivo em crianças (Chappell \& Steitz, 1993; Jegede \& Bamgboye, 1981; Koppitz, 1967), à criatividade (Singer \& Whiton, 1971), à auto-estima (Delatte \& Hendrickson, 1982) e à depressão (Gordon, Lefkowitz \& Tesiny, 1980) entre outros.

O Teste do Desenho da Figura Humana foi desenvolvido por Florence Goodenough na primeira metade do século passado. Trata-se de um instrumento simples e de baixo custo que tem como objetivo a mensuração da inteligência por meio da avaliação da representação da figura humana. Assim, a partir dos itens presentes no desenho, pode-se fazer uma avaliação do desenvolvimento cognitivo da criança que desenha. A utilização desse 
teste baseia-se no pressuposto de que, à medida que a criança cresce, desenvolve também o uso do grafismo e de representações mais evoluídas da figura humana (Sisto, 2000).

Segundo Harris (1963), o Teste do Desenho da Figura Humana de Goodenough é talvez o mais original em sua concepção básica, pela sua brevidade e utilidade geral. Apesar de o teste não ter tempo-limite para a sua realização, a maioria das crianças termina o desenho em menos de dez minutos, havendo crianças que completam o desenho em um ou dois minutos. O Teste do Desenho da Figura Humana é bem recebido pela criança tímida e inibida; pode também ser aplicado a estrangeiros ou iletrados, e não apresenta limitação de idade, inteligência ou habilidade artística (Machover, 1949).

A escala de Goodenough, embora tenha sido desenvolvida com a finalidade de avaliar a inteligência, já foi reexaminada e avaliada como instrumento para o estudo dos transtornos de conduta. O método se apóia, essencialmente, na observação da ausência de partes ou detalhes do corpo, da vestimenta, entre outros, ou da presença de partes definidas com suficiente clareza para quem está avaliando o desenho. Segundo Koppitz (1976), os desenhos de figura humana têm sido utilizados por inúmeros psicólogos ao longo do tempo em que trabalham com crianças. As técnicas utilizadas variam muito, de acordo com o objetivo e função daquele que os aplica. Atualmente, enfocam-se dois tipos de análise possíveis para os DFH. Uma delas é a técnica projetiva, utilizada para desvendar conflitos inconscientes e traços de personalidade. A outra forma de se analisar os desenhos é ressaltando os aspectos da maturação cognitiva. Recentemente, Oliva (2003) realizou uma pesquisa com o objetivo de analisar a produção científica envolvendo o DFH entre os anos de 1998 e 2002. A autora analisou 56 resumos de artigos publicados em periódicos, indexados em cinco bases de dados. Os resultados mostraram que, do total de resumos, 94,6\% utilizou o tipo de trabalho empírico, verificando padronização, precisão e validade, entre outros, enquanto apenas 5,4\% dos estudos se enquadrou no tipo teórico. Quanto ao tipo de avaliação, a autora observou que 50\% utilizaram o DFH como técnica projetiva, 28,6\% como medida de desenvolvimento cognitivo, $14,3 \%$ como projetivo e cognitivo, e $7,1 \%$ não especificaram o tipo de uso. Do total de trabalhos analisados, foi constatado que crianças foram os sujeitos das pesquisas em $35,7 \%$ dos resumos.

Pesquisas relacionando o Teste Gestáltico Visomotor de Bender (TGB) e o DFH podem ser verificadas em todas as décadas a partir de 1940. Por exemplo, Upadhyaya e Sinha (1974) estudaram o TGB e o DFH em pacientes que apresentavam retardo mental. Os resultados indicaram que sujeitos de inteligência mais baixa mostraram mais sinais de dano cerebral no TGB, assim como obtiveram uma idade mental mais baixa no DFH.

Num outro enfoque, Koppitz, Sullivan, Blyth e Shelton (1959) testaram a hipótese de que o TGB e o DFH, administrados no início da primeira série, poderiam predizer a realização escolar no final do ano. Os resultados mostraram que ambos os testes têm a habilidade de predizer a realização escolar, e que esse poder aumenta quando eles são usados juntos como preditores múltiplos. $\mathrm{Na}$ mesma linha, Bandeira e Hutz (1994) investigaram o grau de predição do rendimento escolar na primeira série por meio da aplicação dos testes Desenho da Figura Humana, Bender e Raven. Vários aspectos foram verificados a partir desse estudo. Não houve diferenças significativas entre sexos no desempenho em nenhum dos três testes, assim como não houve evolução dos itens maturacionais e emocionais da amostra no intervalo de oito meses. Em relação à predição do rendimento escolar, os resultados mostraram que os três testes apresentaram correlações significativas, embora apenas o Bender e itens evolutivos do Desenho da Figura Humana tenham contribuído para explicar a variância do rendimento escolar. 
Como foi observado, o Teste Gestáltico Visomotor de Bender e o Desenho da Figura Humana são instrumentos muito pesquisados na área de avaliação psicológica. Eles foram e são utilizados em estudos que abarcam vários aspectos da ciência psicológica. Como mencionado anteriormente, pesquisas realizadas com ambos os testes procuraram verificar até que ponto eles se mostraram preditores do desempenho acadêmico em crianças de séries iniciais quando aplicados numa bateria de testes. Nesse contexto, o objetivo desta pesquisa foi diferente, procurando analisar e relacionar os resultados obtidos por crianças no TGB e no DFH. A hipótese inicial foi que haveria uma correlação negativa entre os testes, ou seja, conforme aumentasse o nível intelectual das crianças a partir do DFH, diminuiria o número de erros apresentados no TGB.

\section{Método}

Participantes

Participaram da pesquisa 312 crianças, sendo 161 (51,6\%) do sexo masculino e 151 (48,4\%) do sexo feminino, de 1a a $4^{a}$ série do ensino fundamental de uma escola pública do interior do Estado de São Paulo. As idades variaram entre sete e dez anos (média 8,62 anos e desvio padrão de 1,12).

Instrumentos

Teste do Desenho da Figura Humana

O Teste do Desenho da Figura Humana propõese a avaliar aspectos cognitivos em crianças. Neste estudo, optou-se por 49 itens a serem analisados segundo o critério de Florence Goodenough, a saber: cabeça, pernas, braços, tronco, tronco mais comprido que largo, ombros, união de braços e pernas, união de braços e pernas na posição correta, pescoço, pescoço continuando a cabeça, olhos, nariz, boca, boca e nariz em duas dimensões, orifícios do nariz, cabelos, cabelos que não excedam a circunferência do crânio, roupas, pelo menos duas peças de vestir, desenho completo sem transparências, quatro peças de vestir bem definidas, vestimenta completa sem incongruências, dedos, número correto de dedos, detalhe dos dedos, polegar em oposição, representação da mão, dedo e braço, articulação braço-cotovelo-ombro, articulação pernajoelhos-cadeira, proporção da cabeça, dos braços, das pernas e dos pés, braços e pernas em duas dimensões, representação de saltos, coordenação motora $\mathrm{A}$, coordenação motora B, coordenação motora na continuação da cabeça, coordenação motora na continuação do tronco, coordenação motora na continuação braços e pernas, coordenação motora na continuação da face, orelhas, posição e proporção correta das orelhas, sobrancelha e pestana, pupilas, proporção do olho, olhar, testa e queixo, e projeção no queixo. A correção foi feita por presença ou ausência dos itens em cada critério, atribuindo-se 1 para presença e 0 para ausência, sendo que a pontuação total por sujeito foi produto da soma total das pontuações de cada item.

Teste Gestáltico Visomotor de Bender

O Teste Gestáltico Visomotor de Bender foi construído para fornecer um índice de maturação perceptomotora e consiste de nove figuras (A, 1, 2, 3, 4, 5, 6, 7 e 8) para serem copiadas da melhor maneira possível, sem qualquer tipo de auxílio mecânico.

Os critérios utilizados foram os de Koppitz, segundo o qual se avaliam quatro quesitos: distorção de forma pelas figuras $A, 1,3,5,6,7$ e 8; integração pelas figuras $A, 2,3,4,5,6$ e 7; rotação pelas figuras $\mathrm{A}, 1,2,3,4,5,7$ e 8, e perseveração pelas figuras 1,2 e 6 . Neste estudo, foram analisados apenas os itens referentes à distorção e integração de forma nas figuras A, 1, 2, 3, 4, 5 e 7. A correção foi feita atribuindo-se um ponto para cada erro cometido pela criança.

Com base na correção e soma dos itens, foram criadas medidas. A distorção de forma, somatório dos pontos atribuídos às figuras analisadas nesse quesito, a integração de forma, somatório dos 
pontos atribuídos às figuras analisadas nesse quesito, e a pontuação geral, somatório das medidas anteriores.

\section{Procedimento}

Após a aprovação do Comitê de Ética da Universidade São Francisco e a assinatura dos responsáveis legais das crianças do Termo de Consentimento Livre e Esclarecido, os instrumentos foram aplicados coletivamente em sala de aula. No Desenho da Figura Humana, foi solicitado às crianças para desenhar uma pessoa numa folha de papel sulfite da melhor forma possível e com o máximo de detalhes, informando-lhes que era permitido o uso da borracha, a qualquer momento, e que o desenho deveria ser feito a lápis.

No caso do Bender, foi solicitado às crianças que copiassem os desenhos apresentados numa folha de papel sulfite da melhor forma possivel, informando que era permitido o uso de borracha a qualquer momento. Entretanto, não foi permitida a utilização de qualquer ajuda mecânica.

\section{Resultados}

Os resultados serão apresentados em três blocos. No primeiro, apresentam-se os dados descritivos referentes ao Desenho da Figura Humana e ao Teste Gestáltico Visomotor de Bender; no segundo, as análises referentes a ambos os testes no que concerne a sexo e idade, e, por fim, a relação entre as informações fornecidas por ambos os testes.

No Desenho da Figura Humana, as crianças apresentaram a média de 24,41 pontos $(d p=6,72)$, com uma pontuação mínima de 8 e máxima de 49 pontos. Verificou-se uma concentração de pontos entre 17 e 30 itens (68\%). A porcentagem de crianças que desenhou entre oito e dezesseis itens foi de $14,4 \%$, e, acima de trinta itens, foi de $17,6 \%$. No caso da distorção de forma no Bender, as pontuações podiam variar entre 0 e 5 pontos. Verificou-se que as pontuações concentraramse em $0,1,2$ e 3 ( $91,7 \%$ das crianças). As pontuações 4 e 5 foram apresentadas por 8,3\% das crianças. A média foi de 1,64, com um desvio padrão de 1,25. Esses dados indicaram que a maioria das crianças apresentou pouca distorção, e casos de distorção acentuada (pontuações 4 e 5) foram verificadas em vinte e seis crianças, ou $8,3 \%$ delas.

Na integração de forma, as pontuações tinham a possibilidade de variar entre 0 e 6 pontos. Verificou-se que as pontuações $\mathrm{SE}$ concentraram em 0 e 1, congregando $83,7 \%$ das crianças estudadas $(n=312)$. As pontuações 2 e 3 foram obtidas por $16,3 \%$ das crianças. A média foi de 0,74 , com desvio padrão de 0,85 . Os dados indicam que a maioria das crianças apresentou boa integração de forma, e casos mais acentuados de não integração (pontuações $2 \mathrm{e}$ 3) foram identificados em cinqüenta e uma crianças. Tendo em vista que a pontuação máxima poderia ser de 6 pontos, pode-se considerar que mesmo as maiores pontuações apresentadas pelas crianças foram baixas.

Com vistas a um indicador mais global, as pontuações de distorção e integração de forma foram somadas, constituindo-se em apenas um indicador denominado pontuação geral. Nesse caso, a pontuação podia variar entre 0 e 11 pontos. Verificou-se sua concentração em 0 , 1,2 e 3 (75,3\% das crianças). As pontuações 4 e 5 foram apresentadas por 20,5\% das crianças, e as pontuações 6 e 7 , por 4,2\%. A média foi de 2,38 , com um desvio padrão de 1,73. Os dados indicam que as crianças apresentaram um desempenho indicativo de boa configuração, tendo em vista que os casos nos quais poderia haver indicação de algum problema (pontuações 6 e 7) foram constatados em treze crianças $(4,2 \%)$. Sendo a pontuação máxima de 11 pontos, pode-se dizer que mesmo as maiores pontuações presentes nos resultados foram baixas.

Com a finalidade de verificar possíveis diferenças de média em relação ao sexo das crianças nos testes avaliados, foi utilizada a prova t de Student, adotando o nível de significância de 0,05 . Os resultados dessa análise encontramse na Tabela 1.
No Desenho da

Figura Humana, foi solicitado às crianças para desenhar uma pessoa numa folha de papel sulfite da melhor forma possivel e com o máximo de detalhes, informando-lhes que era permitido o uso da borracha, a qualquer momento, e que o desenho deveria ser feito a lápis. 
Tabela 1 - Média e desvio padrão por sexo, e valores de $t$ e $p$ nas medidas do DFH e TGB.

\begin{tabular}{|c|c|c|c|c|c|}
\hline & Sexo & Média & $\mathrm{DP}$ & $t$ & $p$ \\
\hline \multirow[t]{2}{*}{ Desenho da Figura Humana } & Masculino & 23,61 & 6,67 & $-2,19$ & 0,02 \\
\hline & Feminino & 25,27 & 6,70 & & \\
\hline \multirow[t]{2}{*}{ Distorção na figura A } & Masculino & 0,29 & 0,46 & 1,91 & 0,05 \\
\hline & Feminino & 0,20 & 0,40 & & \\
\hline \multirow[t]{2}{*}{ Integração na figura A } & Masculino & 0,04 & 0,22 & $-1,86$ & 0,06 \\
\hline & Feminino & 0,11 & 0,31 & & \\
\hline \multirow[t]{2}{*}{ Distorção na figura 1} & Masculino & 0,06 & 0,24 & $-2,44$ & 0,01 \\
\hline & Feminino & 0,15 & 0,35 & & \\
\hline \multirow[t]{2}{*}{ Integração na figura 2} & Masculino & 0,00 & 0,00 & $-1,03$ & 0,30 \\
\hline & Feminino & 0,00 & 0,08 & & \\
\hline \multirow[t]{2}{*}{ Distorção na figura 3} & Masculino & 0,36 & 0,48 & $-1,38$ & 0,16 \\
\hline & Feminino & 0,44 & 0,50 & & \\
\hline \multirow[t]{2}{*}{ Integração na figura 3} & Masculino & 0,33 & 0,47 & $-1,61$ & 0,10 \\
\hline & Feminino & 0,42 & 0,49 & & \\
\hline \multirow{2}{*}{ Integração na figura 4} & Masculino & 0,07 & 0,26 & $-0,77$ & 0,43 \\
\hline & Feminino & 0,09 & 0,30 & & \\
\hline \multirow[t]{2}{*}{ Distorção na figura 5} & Masculino & 0,47 & 0,50 & $-0,89$ & 0,37 \\
\hline & Feminino & 0,52 & 0,50 & & \\
\hline \multirow[t]{2}{*}{ Integração na figura 5} & Masculino & 0,03 & 0,17 & $-0,70$ & 0,48 \\
\hline & Feminino & 0,04 & 0,21 & & \\
\hline \multirow[t]{2}{*}{ Distorção na figura 7} & Masculino & 0,40 & 0,49 & $-0,23$ & 0,81 \\
\hline & Feminino & 0,41 & 0,49 & & \\
\hline \multirow[t]{2}{*}{ Integração na figura 7} & Masculino & 0,13 & 0,34 & $-1,48$ & 0,13 \\
\hline & Feminino & 0,19 & 0,40 & & \\
\hline \multirow[t]{2}{*}{ Distorção de forma } & Masculino & 1,58 & 1,24 & $-0,92$ & 0,35 \\
\hline & Feminino & 1,71 & 1,27 & & \\
\hline \multirow[t]{2}{*}{ Integração de forma } & Masculino & 0,62 & 0,77 & $-2,64$ & 0,00 \\
\hline & Feminino & 0,87 & 0,92 & & \\
\hline \multirow[t]{2}{*}{ Pontuação geral } & Masculino & 2,20 & 1,69 & $-1,97$ & 0,05 \\
\hline & Feminino & 2,58 & 1,75 & & \\
\hline
\end{tabular}

A Tabela 1 evidenciou que o Desenho da Figura Humana, a distorção de forma nas figuras A e 1, a integração de forma geral e a pontuação geral do Bender apresentaram diferenças estatisticamente significativas entre os sexos das crianças ( $t=-2,19, p=0,02 ; t=1,91, p=0,05 ; t=-2,44, p=0,01$; $t=-2,69$ e $p=0,00 ; t=-1,97, p=0,05$ respectivamente). A pontuação média dos meninos foi maior na distorção de forma da figura $\mathrm{A}(0,29, d p=0,46)$, enquanto as meninas obtiveram a média de $0,20(d p=0,40)$. Além disso, evidenciou-se que as meninas obtiveram uma pontuação média maior no Desenho da Figura Humana, na distorção de forma da figura 1, integração de forma geral e pontuação geral do Bender $(25,27, d p=6,70 ; 0,15, d p=0,35 ; 0,87, d p=0,92$ e 2,58, $d p=1,75$ respectivamente), enquanto os meninos obtiveram a média de 23,61 ( $d p=6,67), 0,06(d p=0,24)$, $0,62(d p=0,77)$ e $2,20(d p=1,69)$ nessas medidas.

A Tabela 1 mostrou, ainda, que a integração de forma nas figuras A, 2, 3, 4, 5 e 7 não apresentou diferenças significativas como função do sexo das crianças. Esses mesmos resultados ocorreram na distorção de forma nas figuras 3, 5, 7 e na distorção de forma geral. 
A fim de verificar possíveis diferenças de média como função da idade das crianças, foi utilizada a análise de variância (ANOVA), adotando o nível de significância de 0,05 . Os resultados dessa análise encontram-se na Tabela 2.

Tabela 2 - Valores de $F$ e p obtidos pela análise de variância entre os escores em cada avaliação e a idade das crianças.

\begin{tabular}{|c|c|c|}
\hline & $F$ & $p$ \\
\hline Desenho da Figura Humana & 10,62 & 0,00 \\
\hline Distorção na figura A & 8,36 & 0,00 \\
\hline Integração na figura A & 0,91 & 0,43 \\
\hline Distorção na figura 1 & 3,51 & 0,01 \\
\hline Integração na figura 2 & 1,24 & 0,29 \\
\hline Distorção na figura 3 & 1,60 & 0,18 \\
\hline Integração na figura 3 & 3,26 & 0,02 \\
\hline Integração na figura 4 & 5,00 & 0,00 \\
\hline Distorção na figura 5 & 2,51 & 0,06 \\
\hline Integração na figura 5 & 6,05 & 0,00 \\
\hline Distorção na figura 7 & 18,32 & 0,00 \\
\hline Integração na figura 7 & 4,35 & 0,00 \\
\hline Distorção de forma & 11,00 & 0,00 \\
\hline Integração de forma & 5,31 & 0,00 \\
\hline Pontuação geral & 11,76 & 0,00 \\
\hline
\end{tabular}

Não houve diferenças significativas em relação à idade das crianças estudadas em apenas quatro situações, quais sejam, as medidas integração de forma nas figuras A e 2 e distorção de forma nas figuras 3 e 5 . As outras 11 medidas diferenciaram as idades. Assim, a análise de variância apontou diferenças significativas em relação à idade das crianças nos seguintes itens avaliados: Desenho da Figura Humana $(F=10,62, p=0,00)$, distorção de forma nas figura $A, 1$ e $7(F=8,36$, $p=0,00 ; F=3,51, p=0,01$ e $F=18,32$, $p=0,00$ respectivamente), integração de forma nas figuras $3,4,5$ e $7(F=3,26$, $p=0,02 ; F=5,00, p=0,00 ; F=6,05, p=0,00$ e $F=4,35, p=0,00$ respectivamente) e, na somatória de medidas, distorção de forma geral, integração de forma geral e pontuação geral do Bender $(F=11,00, p=0,00 ; F=5,31$, $p=0,00 \quad$ e $\quad F=11,76, \quad p=0,00$ respectivamente).

Para verificar quais as idades que justificam as diferenças encontradas em cada uma das medidas estudadas, utilizou-se a prova de Tukey. Como resultado, verificou-se que o DFH não se apresentou como um instrumento capaz de mensurar com discriminação o caráter maturacional das crianças, pois, embora o valor de $F$ tenha sido significativo, as idades estudadas não foram separadas.

Deve ser ressaltado que, da mesma forma que no DFH, algumas medidas do TGB, assim como também a distorção de forma, integração de forma e pontuação geral, não separaram as idades estudadas quando aplicada a prova de Tukey, embora os valores de $F$ tenham sido significativos. Isso poderia revelar a necessidade de o sistema de correção empregado ser revisto, com a finalidade de se obter um instrumento capaz de mensurar o caráter maturacional das crianças.

Com a finalidade de evidenciar possíveis relações entre o desempenho das crianças no Desenho da Figura Humana e nas medidas de distorção de forma avaliadas pelas figuras do Teste de Bender, foram correlacionados os dados de ambos os testes para a amostra total, para cada faixa etária e, dentro destas, para os grupos masculino, feminino e geral. Os resultados podem ser visualizados na Tabela 3. Encontram-se ressaltados em negrito os resultados estatisticamente significativos.

Tabela 3 - Coeficientes de correlação de Spearman $(r)$ e valores de $p$ entre o Desenho da Figura Humana e a distorção de forma em cada uma das figuras. 


\begin{tabular}{|c|c|c|c|c|c|c|c|c|c|c|c|}
\hline & & \multicolumn{10}{|c|}{ Teste Gestáltico Visomotor de Bender } \\
\hline & & \multicolumn{2}{|c|}{ DFa } & \multicolumn{2}{|c|}{ DF1 } & \multicolumn{2}{|c|}{ DF3 } & \multicolumn{2}{|c|}{ DF5 } & \multicolumn{2}{|c|}{ DF7 } \\
\hline & & $r$ & $p$ & $r$ & $p$ & $r$ & $p$ & $r$ & $p$ & $r$ & $p$ \\
\hline & Total & $-0,25$ & 0,00 & 0,00 & 0,96 & 0,00 & 0,92 & 0,09 & 0,09 & $-0,29$ & 0,00 \\
\hline \multirow{3}{*}{$\begin{array}{c}7 \\
\text { anos }\end{array}$} & Masculino & 0,05 & 0,71 & $-0,00$ & 0,99 & 0,19 & 0,21 & $-0,07$ & 0,61 & $-0,19$ & 0,21 \\
\hline & Feminino & $-0,22$ & 0,30 & $-0,18$ & 0,40 & $-0,18$ & 0,38 & 0,01 & 0,93 & $-0,26$ & 0,21 \\
\hline & Geral & $-0,05$ & 0,64 & $-0,05$ & 0,68 & 0,09 & 0,44 & $-0,04$ & 0,74 & $-0,23$ & 0,05 \\
\hline \multirow{3}{*}{$\begin{array}{c}8 \\
\text { anos }\end{array}$} & Masculino & $-0,28$ & 0,06 & $-0,22$ & 0,13 & $-0,15$ & 0,30 & $-0,02$ & 0,89 & $-0,27$ & 0,06 \\
\hline & Feminino & $-0,27$ & 0,11 & 0,06 & 0,72 & $-0,39$ & 0,01 & 0,14 & 0,39 & $-0,21$ & 0,20 \\
\hline & Geral & $-0,27$ & 0,01 & $-0,04$ & 0,67 & 0,10 & 0,35 & 0,05 & 0,61 & $-0,22$ & 0,03 \\
\hline \multirow{3}{*}{$\begin{array}{c}9 \\
\text { anos }\end{array}$} & Masculino & $-0,33$ & 0,07 & 0,14 & 0,45 & 0,12 & 0,53 & 0,17 & 0,36 & $-0,13$ & 0,51 \\
\hline & Feminino & $-0,26$ & 0,08 & $-0,06$ & 0,65 & $-0,12$ & 0,43 & 0,20 & 0,18 & $-0,32$ & 0,03 \\
\hline & Geral & $-0,31$ & 0,00 & 0,02 & 0,83 & $-0,02$ & 0,85 & 0,22 & 0,06 & $-0,21$ & 0,06 \\
\hline \multirow{3}{*}{$\begin{array}{c}10 \\
\text { anos }\end{array}$} & Masculino & $-0,20$ & 0,16 & 0,17 & 0,25 & $-0,15$ & 0,30 & 0,16 & 0,27 & $-0,21$ & 0,16 \\
\hline & Feminino & $-0,08$ & 0,56 & $-0,06$ & 0,64 & $-0,04$ & 0,77 & 0,01 & 0,91 & $-0,22$ & 0,13 \\
\hline & Geral & $-0,13$ & 0,20 & $-0,00$ & 0,95 & $-0,10$ & 0,32 & 0,08 & 0,43 & $-0,21$ & 0,03 \\
\hline
\end{tabular}

Dfa=Distorção de forma na figura A / Df1=Distorção de forma na figura 1 / Df3=Distorção de forma na figura 3 / Df5=Distorção de forma na figura 5 / Df7=Distorção de forma na figura 7.

Como mostra a Tabela 3, houve correlação negativa e significativa entre o Desenho da Figura Humana e a distorção de forma nas figuras A e $7(r=-0,25, p=0,00$ e $r=-0,29, p=0,00$ respectivamente) de forma geral, ou seja, quando consideradas todas as crianças, independentemente do sexo e idade. Verificou-se, ainda, uma correlação negativa e significativa entre o DFH e distorção de forma nas figuras A e 7 ( $r=-0,27, p=0,01$ e $r=-0,22, p=0,03$ respectivamente) para 8 anos, independentemente do sexo. Houve, ainda, correlação negativa entre o DFH e a distorção de forma na figura 7 para os sete e dez anos, independentemente do sexo das crianças, 7 ( $r=-0,23, p=0,05$ e $r=-0,21, p=0,03$ respectivamente), e, para as crianças de nove anos, do sexo feminino, $7(r=-0,32, p=0,03)$. Verificou-se também uma correlação negativa para as crianças de nove anos na distorção de forma na figura $\mathrm{A}(r=-0,31, p=0,00)$. A distorção de forma, na figura 3 , apresentou apenas uma correlação negativa e significativa para as crianças de oito anos do sexo masculino $7(r=-0,39, p=0,01)$. A distorção de forma, nas figuras 1 e 5, não apresentou correlações significativas com nenhuma das idades e sexo das crianças estudadas no Desenho da Figura Humana.

Quando feita uma análise qualitativa mais aprofundada da Tabela 3, pode-se verificar que, das 65 correlações possíveis, apenas nove se mostraram significativas, sendo que, dessas nove, duas se referiram às pontuações totais na distorção de forma na figura A e na distorção de forma na figura 7. Em relação à integração de forma, os dados referentes às correlações podem ser visualizados na Tabela 4. 
Tabela 4 - Coeficientes de correlação de Spearman $(r)$ e valores de $p$ entre o Desenho da Figura Humana e a integração em cada uma das figuras.

\begin{tabular}{|c|c|c|c|c|c|c|c|c|c|c|c|c|c|}
\hline & \multicolumn{12}{|c|}{ Teste Gestáltico Visomotor de Bender } \\
\hline & & \multicolumn{2}{|l|}{ IA } & \multicolumn{2}{|c|}{12} & \multicolumn{2}{|c|}{ I3 } & \multicolumn{2}{|c|}{14} & \multicolumn{2}{|c|}{15} & \multicolumn{2}{|c|}{17} \\
\hline & & $r$ & $p$ & $r$ & $p$ & $r$ & $p$ & $r$ & $p$ & $r$ & $p$ & & $p$ \\
\hline \multicolumn{2}{|c|}{ Total } & $-0,03$ & 0,59 & $-0,04$ & 0,42 & $-0,17$ & 0,00 & $-0,10$ & 0,06 & 0,10 & 0,06 & $-0,20$ & 0,00 \\
\hline \multirow{3}{*}{$\begin{array}{c}7 \\
\text { anos }\end{array}$} & M & 0,01 & 0,94 & $* * *$ & $* * *$ & $-0,06$ & 0,67 & $-0,22$ & 0,14 & $-0,04$ & 0,79 & $-0,14$ & 0,37 \\
\hline & $\mathrm{F}$ & 0,05 & 0,78 & $-0,14$ & 0,50 & $-0,22$ & 0,30 & $-0,23$ & 0,27 & $* * *$ & $* * *$ & $-0,33$ & 0,10 \\
\hline & G & 0,05 & 0,66 & $-0,03$ & 0,77 & $-0,07$ & 0,55 & $-0,15$ & 0,20 & $-0,05$ & 0,64 & $-0,15$ & 0,22 \\
\hline \multirow{3}{*}{$\begin{array}{c}8 \\
\text { anos }\end{array}$} & $M$ & $* * *$ & $* * *$ & $* * *$ & $* * *$ & $-0,33$ & 0,02 & 0,06 & 0,68 & $* * *$ & $* * *$ & 0,02 & 0,89 \\
\hline & $\mathrm{F}$ & 0,10 & 0,56 & $* * *$ & $* * *$ & $-0,24$ & 0,16 & $-0,04$ & 0,77 & $* * *$ & $* * *$ & $-0,20$ & 0,24 \\
\hline & G & 0,09 & 0,42 & $* * *$ & $* * *$ & $-0,27$ & 0,01 & 0,01 & 0,92 & $* * *$ & $* * *$ & $-0,08$ & 0,47 \\
\hline \multirow{3}{*}{$\begin{array}{c}9 \\
\text { anos }\end{array}$} & $M$ & $-0,01$ & 0,94 & $* * *$ & $* * *$ & $-0,12$ & 0,51 & 0,18 & 0,34 & $* * *$ & $* * *$ & $-0,15$ & 0,43 \\
\hline & $\mathrm{F}$ & $-0,16$ & 0,27 & $* * *$ & $* * *$ & $-0,23$ & 0,11 & $-0,24$ & 0,10 & $-0,08$ & 0,57 & $-0,19$ & 0,20 \\
\hline & G & $-0,09$ & 0,42 & $* * *$ & $* * *$ & $-0,15$ & 0,18 & $-0,10$ & 0,40 & $-0,04$ & 0,70 & $-0,17$ & 0,13 \\
\hline \multirow{3}{*}{$\begin{array}{c}10 \\
\text { anos }\end{array}$} & $M$ & 0,02 & 0,88 & $* * *$ & $* * *$ & $-0,05$ & 0,73 & 0,17 & 0,25 & 0,21 & 0,16 & $-0,37$ & 0,01 \\
\hline & $F$ & $-0,11$ & 0,45 & $* * *$ & $* * *$ & $-0,10$ & 0,48 & $* * *$ & $* * *$ & 0,12 & 0,34 & $-0,20$ & 0,16 \\
\hline & G & $-0,05$ & 0,60 & $* * *$ & $* * *$ & $-0,08$ & 0,43 & 0,13 & 0,21 & 0,16 & 0,12 & $-0,27$ & 0,00 \\
\hline
\end{tabular}

la =Integl ação de forma na figura A / I2=Integração de forma na figura $2 / 13=$ Integração de forma na figura $3 /$ $14=$ Integração de forma na figura $4 / 15=$ Integração de forma na figura $5 / 17=$ Integração de forma na figura 7 . ***=Não pôde ser computado porque a variável é constante.

A Tabela 4 evidenciou que houve correlação negativa e significativa entre o Desenho da Figura Humana e a integração de forma nas figuras 3 e $7(r=-0,17, p=0,00$ e $r=-0,20, p=0,00$ respectivamente) de forma geral, ou seja, quando consideradas todas as crianças independentemente do sexo e idade. Verificou-se, ainda, uma correlação negativa e significativa entre o DFH e integração de forma na figura 3 para as crianças de oito anos do sexo masculino e de forma geral, ou seja, independentemente do sexo $(r=-0,33, p=0,02$ e $r=-0,27, p=0,01$ respectivamente). Houve, ainda, correlação negativa e significativa entre o DFH e a integração de forma na figura 7 para as crianças de dez anos do sexo masculino e as crianças dessa idade de forma geral ( $r=-0,37, p=0,01$ e $r=-0,27, p=0,00$ respectivamente). A integração de forma avaliada pelas figuras A, 2, 4 e 5 não apresentou correlações significativas com nenhuma das idades e sexo das crianças estudadas no Desenho da Figura Humana.

Da mesma forma que na distorção de forma, das 78 correlações possíveis na integração de forma, apenas seis se mostraram significativas, o que poderia estar evidenciando a necessidade de um novo sistema de correção para o TGB. A seguir, foram estudadas as correlações referentes a distorção total, integração total e pontuação total do Bender. Os resultados podem ser visualizados na Tabela 5. 
Tabela 5 - Coeficientes de correlação de Pearson $(r)$ e valores de $p$ entre o Desenho da Figura Humana e as medidas produto da soma de itens.

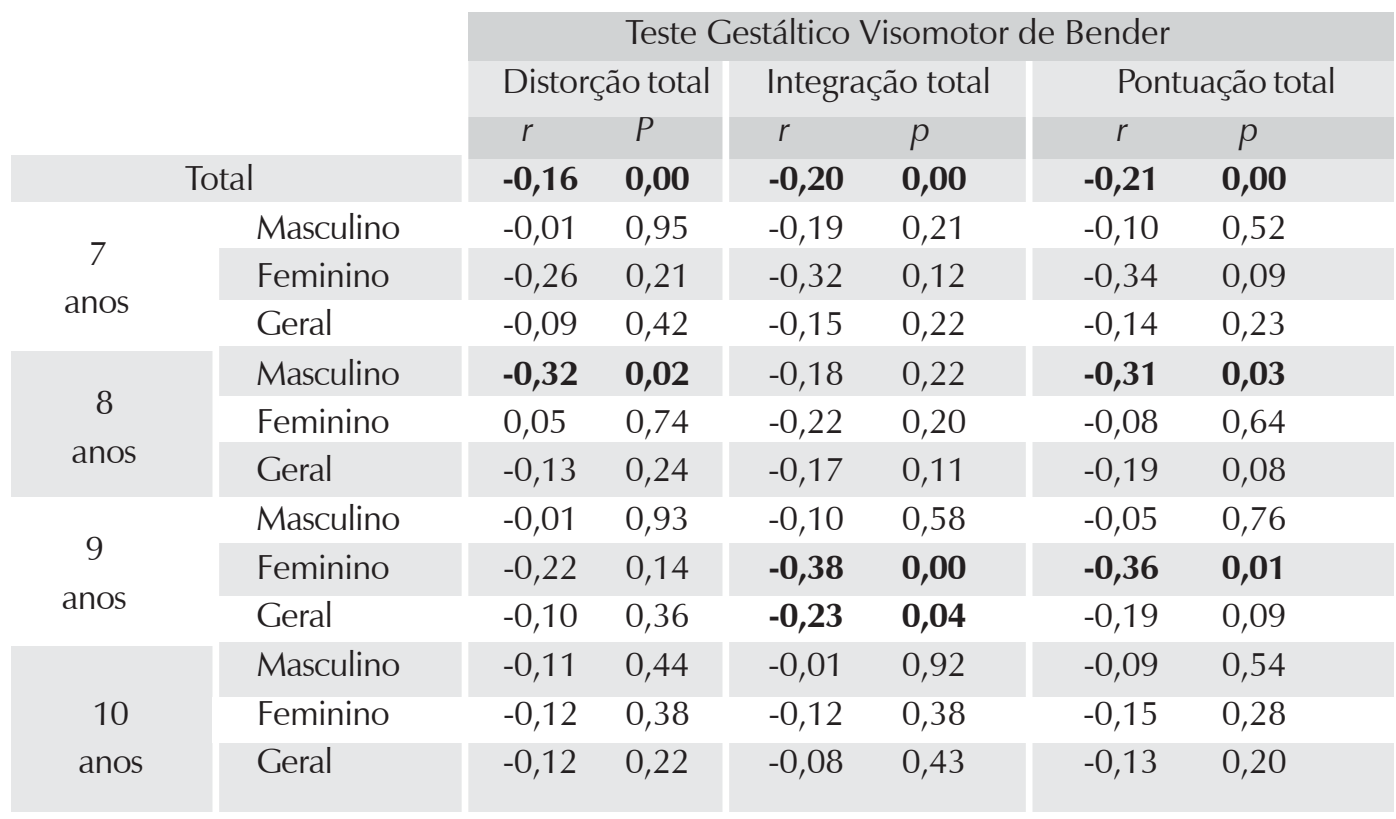

Como mostra a Tabela 5, houve correlação negativa e significativa entre o Desenho da Figura Humana e a distorção e integração de forma geral, assim como também na pontuação total do Bender de forma geral $(r=-0,16, p=0,00 ; r=-0,20, p=0,00$ e $r=-0,21, p=0,00$ respectivamente). Verificou-se também uma correlação negativa e significativa entre o DFH e a distorção de forma e pontuação total do Bender para as crianças de oito anos do sexo masculino ( $r=-0,32, p=0,02$ e $r=-0,31, p=0,03$ respectivamente). A pontuação total do Bender apresentou, ainda, correlação negativa para as crianças de nove anos do sexo feminino $(r=-$ $0,36, p=0,01)$. A integração de forma total também apresentou correlação para as crianças de nove anos do sexo feminino e crianças dessa mesma idade de forma geral $(r=-0,38, p=0,00$ e $r=-0,23, p=0,04$ respectivamente).

No caso das pontuações totais, pode ser evidenciado que, das 36 correlações possíveis, além das correlações das pontuações totais, apenas cinco se mostraram estatisticamente significativas. Esse fato pode sugerir que, embora o TGB se apresente medindo parte do mesmo construto que o DFH, outros critérios poderiam ser utilizados na correção de cada uma das suas figuras com a finalidade de aprimorar o caráter maturacional que o teste se propõe a medir.

\section{Discussão e conclusões}

O presente trabalho objetivou analisar qual a relação entre o desempenho no Teste Gestáltico Visomotor de Bender e o Desenho da Figura Humana (utilizando o sistema de Goodenough). Assim, verificou-se que as crianças estudadas apresentaram níveis baixos em distorção de forma e em integração da forma avaliada pelo TGB. Quanto ao DFH, as crianças, na sua maioria, apresentaram uma quantidade de itens esperados para as idades estudadas (Harris, 1963). 
Aprofundando as análises, verificaram-se possíveis diferenças quanto ao sexo e às idades das crianças estudadas. Em relação ao sexo, houve diferenças significativas no $\mathrm{DFH}$, sendo que as meninas desenharam uma quantidade maior de detalhes que os meninos, indo esses resultados ao encontro do proposto por Harris (1963), segundo o qual há uma predominância de detalhes nos desenhos em crianças do sexo feminino.

Quanto ao TGB, apenas três dos 11 itens avaliados diferenciaram os sexos, assim como também a somatória dos itens de integração de forma e a pontuação geral do teste. Em relação aos itens avaliados de forma separada, verificou-se que duas figuras avaliadas na distorção de forma mostraram diferenças significativas, enquanto apenas uma, em integração de forma, mostrou tais diferenças.

Em relação à idade, se, por um lado, houve diferenças significativas no DFH evidenciados pelos valores significativos de $F$, corroborando, assim, os dados da literatura, que evidenciam maior quantidade de detalhes desenhados à medida que a idade das crianças aumenta (Chappell \& Steitz, 1993; Harris, 1963; Jegede \& Bamgboye, 1981), por outro, tais diferenças foram muito pequenas, e, quando foi aplicada, a prova de Tukey mostrou que o DFH não separou as idades das crianças estudadas, evidenciando que tal teste não estaria avaliando com discriminação o caráter maturacional. Quanto ao TGB, em quatro itens avaliados individualmente, não houve efeito significativo da idade, enquanto que, em outros sete itens, assim como a integração de forma, distorção de forma e pontuação geral no teste, tal efeito foi significativo. Esses resultados corroboraram os de Koppitz (1959, 1989), nos quais a autora ressalta que, à medida que o desenvolvimento cognitivo da criança se processa, a pontuação no TGB tende a diminuir.

Quando relacionados ambos os testes, as figuras A, 3 e 7 do TGB apresentaram correlações negativas e significativas com o DFH, enquanto as figuras 1, 2, 4 e 5 não mostraram nenhuma relação significativa com o Teste do Desenho da Figura Humana. Quando comparado o DFH com as pontuações totais no TGB, independentemente de sexo e idade, verificaram-se correlações negativas e significativas nas três medidas do Bender, quais sejam, distorção da forma, integração da forma e pontuação total no teste. Embora as correlações tenham sido baixas $(-0,16$ até $-0,21)$, os dados confirmaram a hipótese inicial do estudo, segundo o qual quanto melhor o desempenho das crianças no DFH, menor será a pontuação no TGB, porém, a comunalidade foi de apenas $4 \%$, sugerindo ainda que os testes podem estar medindo construtos diferentes.

Além disso, esta pesquisa vai ao encontro do trabalho realizado por Upadhyaya e Sinha (1974), pois as crianças que apresentaram um pior desempenho no TGB apresentaram também uma idade mental mais baixa no DFH. Embora esses dados não possam ser tomados como conclusivos, eles indicam uma tendência que vem sendo verificada pela literatura científica ao longo das décadas. Assim, novas pesquisas se fazem necessárias, com a finalidade de trazer novas informações para a avaliação cognitiva de crianças por meio de métodos fáceis e válidos para a realidade brasileira. 
Fabián Javier Marín Rueda - Universidade São Francisco

Psicólogo, Mestre em Psicologia e doutorando em Avaliação Psicológica pela Universidade São Francisco - Itatiba. Bolsista da CAPES.

Daniel Bartholomeu - Universidade São Francisco

Mestrando em Avaliação Psicológica pela Universidade São Francisco Itatiba. Bolsista da CAPES.

\section{Fermino Fernandes Sisto - Universidade São Francisco}

Doutor pela Universidad Complutense de Madrid, Livre-docente pela

Unicamp e docente do Curso de Psicologia e do Programa de Pós-graduação

Stricto Sensu em Psicologia da Universidade São Francisco - Itatiba.

Contato com primeiro autor.

Av. Expedicionários Brasileiros 270, ap.42 Centro, Itatiba-SP

CEP: 13256-400 Fone: (11) 4538-6797

E-mail: marinfabian@yahoo.com.br 
ALVES, I. C. B. Instrumentos Disponíveis no Brasil para Avaliação da Inteligência. In: R. Primi (org.) Temas em Avaliação Psicológica, Campinas: IBAP, 2002, pp.80-102.

BACHARA, G. H., ZABA, J. N. \& RASKIN, L. M. Human Figure Drawings and LD Children. Academic Therapy, 11(2), pp.217-222, 1975-1976.

BAMBAREN, V. C. La Prueba de la Gestalt de Bender en Esquizofrenicos. Revista de Psicología General y Aplicada, 13, pp.481-509, 1958.

BANDEIRA, D. R. \& HUTZ, C. S. A Contribuição dos Testes DFH, Bender e Raven na Predição do Rendimento Escolar na Primeira Série. Psicologia: Teoria e Pesquisa, 10(1), pp. 59-72, 1994.

BEUTLER, L. Parameters in the Prediction of Police Officer Performance. Professional Psychology: Research and Practice, 16(2), pp.324-335, 1985.

CHAPPELL, P. A. \& STEITZ, I. A. Young Children`s Human Figure Drawing and Cognitive Development. Perceptual and Motor Skills, 76(2), pp. 611-617, 1993.

CLAWSON, A. Bender Infantil: Manual de Diagnóstico Clínico Porto Alegre, Artes Médicas, 1980.

CURTY, R. M. L. Realidade Empírica da Pesquisa Brasileira em Técnicas Projetivas. Arquivos Brasileiros de Psicologia, 37(2), pp.156-167, 1985.

DELATTE, J. G. \& HENDRICKSON, N. J. Human Figure Drawings Size as a Measure of Self-esteem. Journal of Personality Assessment 46(6), pp.603-606, 1982.

GORDON, N.; LEFKOWITZ, M. M. \& TESINY, E. P. Childhood Depression and the Draw-a-Person Test. Psychological Reports, 47(1), pp. 251-257, 1980.

GOUNARD, B. R. \& PRAY, R. C. Human Figure Drawings of Learning Disables and Normal Children at three Age Levels. Perceptual and Motor Skills, 40(3), p. 914, 1975.

HARRIS, D. B. El Test de Goodebough: Revisión, Ampliación y Actualización. Barcelona: Paidós, Psicometria e Psicodiagnóstico 1963.

HOLMES, C. B., DUNGAN, D. S. \& MEDLIN, W. J. Reassessment of Inferring Personality Traits from Bender-Gestalt Drawing Styles. Journal of Clinical Psychology, 40(5), 1241-1243.

JEGEDE, R. O. \& BAMGBOYE, E. A. Intellectual Maturity in Nigerian Primary School Children. South African Journal of Psychology, 11(2), pp.87-89, 1981.

JING, l.: YUAN, C. \& LIU, J. Study of Human Figure Drawings in Learning Disabilities. Chinese Mental Health Journal, 13(3), pp.133-134, 1999.

KAHIILL, S. Human Figure Drawing in Adults: an Update of the Empirical Evidence, 1967-1982. Canadian Psychology, 25(4), pp.269-292, 1984.

KOPPITZ, E. M., SULLIVAN, J., BLYTH, D. D. \& SHELTON, J. Prediction of First Grade School Achievement with the Bender Gestalt Test and Human Figure Drawing. Journal of Clinical Psychology, 15, pp.164168,1959 .

KOPPITZ, E. M. Expected and Exceptional Items on Human Figure Drawing and IQ Scores of Children Age 5 to 12. Journal of Clinical Psychology, 23(1), pp.81-83, 1967.

El Dibujo de la Figura Humana en los Niños. Editorial Guadalupe. Buenos Aires, 1976.

O Teste Gestáltica Bender para Crianças. Porto Alegre, Artes Médicas, 1989

MACHOVER, K. Personality Projection in the Drawing of the Human Figure. Springfield: Charles C. Thomas, 1949.
MAREK, K.; ZAJAC, N. M.; ROLA, E. \& WOCKA M. T. Health Effect of Exposure to Metallic Mercury Vapours in Workers Engaged in the Production of Chlorine and Acetic Aldehyde: I. Evaluation of the General Health Condition. Medycyna Pracy, 46(2), pp.101-109, 1995.

MATCHABELY, K. \& BERTRAND, R. Quelques Considerations Pratiques sur l'Application du Test Moteur de Structuration Visuelle de Bender en Clinique Psychiatrique. Revue de Psychologie Appliquée, 3, pp.326-332, 1953.

MURRAY, E. J. \& ROBERTS, F. J. The Bender-Gestalt Test in a Patient Passing through a Brief Manic-depressive Cycle. United States Armed Forces Medical Journal, 7, pp.1206-1208, 1956.

NGUYEN, K. C. Les Épreuves Graphiques: Methode d'ÉvaluationMethode d'Investigation. Bulletin de Psychologie, 45(406), pp. 449$455,1992$.

NORONHA, A. P. P. Análise de Coeficientes de Testes de Inteligência. Psico, 32(2), pp.73-86, 2001.

Os Problemas mais Graves e mais Freqüentes no Uso dos Testes Psicológicos. Psicologia: Reflexão e Crítica, 15(1), pp.135142, 2002.

NORONHA, A. P. P.; OLIVEIRA, A. F.; COBÊRO, C.; PAULA, L. M. CANTALICE, L. M.; GUERRA, P. B. C.; MARTINS, R. M. M. \& FILIZATTI, R. Instrumentos Psicológicos mais Conhecidos por Estudantes do Sul de Minas Gerais. Avaliação Psicológica, 1(2), pp.151-158, 2002.

OKASHA, A. Academic Difficulty among Male Egyptian University Students: II. Associations with Demographic and Psychological Factors. British Journal of Psychiatry, 146, pp.144-150, 1985

OLIVA, D. V. Cinco Anos de Produção Científica do DFH: Análise das Publicações entre 1998 e 2002. In: Resumos de Comunicação Científica da XXXIII Reunião Anual da Sociedade Brasileira de Psicologia. Belo Horizonte, p.396, 2003.

POLLAK, J. M. Human Figure Drawing Performance of LD Children: Research and Clinica Perspectives. Learning Disability Quarterly, 9(3), pp.173-181, 1986.

PRADO, W. M.; PEYMAN, D. A. \& LACEY, O. L. A Validation Study of Measures of Flattened Affect on the Bender-Gestalt Test. Journal of Clinical Psychology, 16(4), pp.435-438, 1960.

SISTO, F. F. Relationships of the Piagetian Cognitive Development to Human Figure Drawing. Child Study Journal, 30(4), pp.225-232, 2000.

SANGSTER, G.; ROGERS, B. \& SEARIGHT, H. R. The Validity of Hutt's Bender-Gestalt Scoring System for Hostility: Correlation with the Interpersonal Behavior Survey's Aggression Scales. Psychology: a Journal of Human Behavior, 30(2), pp.22-29, 1993

SINGER, D. L. \& WHITON, M. B. Ideational Creativity and Expressive Aspects of Human Figure Drawing in Kindergarten-age Children. Developmental Psychology, 4(3), pp.366-369, 1971.

STEWART, H. \& CUNNINGHAM, S. A Note on Scoring Recalled Figures of the Bender Gestalt Test Using Psychotics, Non-psychotics and Controls. Journal of Clinical Psychology, 14, pp.207-208, 1958.

SUNDBERG, N. D. The Practice of Psychological Testing in Clinical Services in the United States. American Psychologist, 16, pp.79-83, 1961.

TAMKIN, A. S. The Effectiveness of the Bender-Gestalt in Differential Diagnosis. Journal of Consulting Psychology, 21, pp.355-357, 1957.

UPADHYAYA, S. \& SINHA, A. K. Some Findings on Psychodiagnostic Test with Young Retarded Adults. Indian Journal of Clinica Psychology, 1(2), pp.73-79, 1974.

\section{Referências}

